\title{
Pregnancy Rate after Embryo Transfer on Day 5 and Day 6 in IVF. A Systematic Review and Meta-Analysis
}

\author{
Hassan S. Abduljabbar ${ }^{1,2}$, H. Hashim², M. Gehad ${ }^{2}$, Heba E. Hashish ${ }^{2}$, A. Algaradi ${ }^{2}$ \\ ${ }^{1}$ Department Obstetrics and Gynecology, Medical College, King Abdulaziz University, Jeddah, Makkah, Saudi Arabia \\ ${ }_{2}^{2}$ Jeddah Fertility Center at the Department of Obstetrics and Gynaecology, Dr. Erfan Bagedo General Hospital Saudi Arabia, \\ Jeddah, KSA \\ Email: *profaj17@yahoo.com, HHashim@ebgh.sa, hhashimh@aol.com, Mgehad72@yahoo.com, \\ Dr.hebayahashisha@yahoo.com,Dr.Aisha-ghaleb@hotmail.com
}

How to cite this paper: Abduljabbar, H.S., Hashim, H., Gehad, M., Hashish, H.E. and Algaradi, A. (2021) Pregnancy Rate after Embryo Transfer on Day 5 and Day 6 in IVF. A Systematic Review and Meta-Analysis. Advances in Reproductive Sciences, 9, 97-105. https://doi.org/10.4236/arsci.2021.91010

Received: November 9, 2020

Accepted: January 25, 2021

Published: January 28, 2021

Copyright (c) 2021 by author(s) and Scientific Research Publishing Inc. This work is licensed under the Creative Commons Attribution International License (CC BY 4.0).

http://creativecommons.org/licenses/by/4.0/

(c) (i) Open Access

\begin{abstract}
Study Question: The question: is there any difference in pregnancy rate between embryo transfer day 5 and day 6 in IVF? What Is Known Already? Blastocyst transfer is increasingly popular in assisted reproductive technology (ART) centers today. Very few articles concentrate on comparing Day 5 and Day 6 embryo transfer with conflict results. Objective, Study Design: Systematic review and meta-analysis of published controlled studies. Searches conducted from 2001-2020 on PubMed. Medline, EMBASE, and ISI Web of Science Electronic database is used to collect data, using the following search terms: blastocyst, Day 5, Day 6, embryo transfer (E.T.) and pregnancy rate. Materials, Setting, Methods: A total of 6 full-text articles preselected from 211 references, based on title and abstract. Two independent reviewers performed data selection and extraction according to the Preferred Reporting Items for Systematic Reviews and Meta-Analysis statement (PRISMA). This systematic review was conducted according to (PICO) standard. Random-effect meta-analysis performed on all data (overall analysis). Results and the Role of Chance: Data from 6 relevant articles were extracted and integrated into the meta-analysis that reported clinical pregnancy rate (CPR) as an outcome in 5640 cycles, 2274 cycle had embryo transfer at day 6 and the control was 3366 cycle had embryo transfer day 5 showed a significantly higher clinical pregnancy rate following Day 5 embryo transfer compared with Day 6 embryo transfer with odd ratio and 95\% confidence limit 0.73 (0.66 $0.82) p$ value $<0.000$. Sensitivity analysis led to similar results and conclusions. Limitations, Reasons for Caution: The validity of meta-analysis results depends mainly on the quality and the number of published studies available.
\end{abstract}


Indeed, this meta-analysis included no randomized controlled trial (RCT). Wider Implications of the Findings: In regards to the results of this original meta-analysis, ART practitioners should preferably transfer D5 rather than D6 blastocysts. Further RCTs are needed to address the question of whether D6 embryos should be transported.

\section{Keywords}

Day 5, Day 6, IVF, Blastocyst, Clinical Pregnancy Rate

\section{Introduction}

\section{Assisted reproductive technology (ART)}

The most commonly used ART procedures are in vitro fertilization (IVF) and intracytoplasmic sperm injection (ICSI) which start with patient's selection such as women with damaged or blocked fallopian tubes, or infertility due to male factor including decreased sperm count or sperm motility, women with ovulation disorders, and unexplained infertility [1]. The IVF \& ICSI and embryo transfer process start by controlled hyperstimulation to stimulate multiple oocytes productions [1].

This can be monitored by hormonal level and transvaginal ultrasound [2]. Oocytes retrieved through a micro-invasive procedure that uses transvaginal ultrasound to guide a needle to retrieved oocytes [3]. The male asked to produce a sample of sperm [4]. In a process called insemination, the sperm and eggs are mixed and stored in a laboratory dish to encourage fertilization [5]. In some cases of poor semen parameters, intracytoplasmic sperm injection (ICSI) may use where a single sperm is injected directly into the oocytes in an attempt to achieve fertilization. Cell division is taking place and monitored on a daily basis.

The fertilized ovum is now considered embryos [6]. One of the most critical steps is embryo transfer procedure which is usually occurred three to five days following Oocytes pickup [7]. Embryos that develop to the expanded blastocyst stage at embryo transferred on day 5 are likely to have an implantation rate more than embryo transfer on Day 6 [8]. The rate of development to the expanded blastocyst stage (Day five and Day 6) is associated with higher implantation rates than of younger cleavage stage embryos being related to viability [10].

The aim is to identify if there is any difference in pregnancy rate between embryo transfer day 5 and day 6 (Review and Meta-analysis).

\section{Materials and Methods}

(Systematic review and meta-analysis) of published retrospective studies.

Information sources and search strategy.

Conducted from January 2001-January 2020 on PubMed. Medline, EMBASE, 
ISI Web of Science Electronic database used to collect data. Using the following search term: blastocyst, Day 5, Day 6, embryo transfer (E.T.) and pregnancy rate.

\section{Literature search strategy and eligibility criteria}

Studies not published as full manuscripts in peer-reviewed journals were not considered for this review since they cannot adequately be evaluated for their design and quality. This meta-analysis conducted and reported according to the Preferred Reporting Items for Systematic Reviews and Meta-Analysis statement (PRISMA), and (PICO) standard. The criteria for inclusion/exclusion of studies established before the literature search.

A study considered eligible only if the researchers applied a policy (day five and day six embryo transfer). Moreover, the reference lists of the identified studies were reviewed to determine any other new eligible studies. Only studies published in English were eligible for this study.

Two independent authors (AJ, MG) searched PubMed. Medline, EMBASE, and S.I. Web of Science Electronic database retrospective studies in participants that fulfilled the following with Keywords used was "day 5, day 6, embryo transfer" in the title, abstract or index term fields.

Relevant eligible literature was also scanned through cross-references of identification in the reference lists within both original and review articles. For estimation of pregnancy rate, we excluded studies with: letters to the editor, case reports, editorials; or the full data was not accessible even after a request from the corresponding author.

Methodological quality assessment, Data collection, and Sensitivity analysis

The data from eligible studies extracted and assessed by two independent authors (Aj, MG) and an additional author checked these according to the standard protocol.

The following variables collected from the eligible studies: Study name, publication year, study design, number of cycles, odds ratio, 95\% CL lower, and 95\% CL upper, and p value (Table 1 ) and (Table 2 ).

To assess the impact of the weight of the study, sensitivity analyses were performed by visual inspection of the forest plot. Therefore, the meta-analysis was rerun in the first step by step guide Pooled effect sizes were deemed statistically significant at $P<0.05$. In addition to computed estimates of between-study variance, the statistical heterogeneity across the studies was calculated by chi-square statistic, and the value of $\mathrm{I} 2$ statistic judged inconsistency. An $P$ value of over $50 \%$ indicated substantial heterogeneity. Each outcome was analyzed independently [9].

Odds Ratios (O.R.s) with two-sided 95\% CI were estimated for dichotomous outcomes using a random-effect model. Although we analyzed the data using both the fixed effect models and random-effect models, results in the text are only reported from random-effect models due to underlying heterogeneity in the studies. 
Table 1. Characteristics of included studies.

\begin{tabular}{llllll}
\hline Study & Year & Journal & design & \# cycle D6 & \# cycles D5 \\
\hline Bruce Shapiro & 2001 & F\&S. $^{10}$ & Retrosp & 65 & 118 \\
Jigal Haas & 2016 & J Ass RG $^{11}$ & Retr-coho & 254 & 537 \\
V. Poulsen & 2017 & Hum Re $^{12}$ & Retr-coho & 368 & 334 \\
Samer Tannus & 2018 & HumRe $^{13}$ & Retr-coho & 126 & 441 \\
Lucile Ferreux & 2018 & HumRe $^{14}$ & Retr-coho & 944 & 353 \\
Bruce Shapiro & 2019 & Zygote $^{15}$ & Retrosp & 517 & 1583 \\
Total & & & & 2274 & 3366 \\
\hline
\end{tabular}

Retrosp = Retrospective, Retr-coho = Retrospective coherent, $\#=$ Number, D6 = Day 6, D5 = Day 5.

Table 2. Illustrate the weight in \%, and odd ratio 95\% confidence limit (lower and upper) and $\mathrm{p}$ value.

\begin{tabular}{llllll}
\hline Study & Weight & Odd. ratio & lower & Upper & $P$-value \\
\hline Bruce Shapiro, & 2.9 & 0.33 & 0.17 & 0.62 & 0.000 \\
Jigal Haas & 11.2 & 0.63 & 0.46 & 0.86 & 0.004 \\
V. Poulsen & 16.2 & 0.25 & 0.18 & 0.34 & 0.000 \\
Samer Tannus, & 5.5 & 1.09 & 0.71 & 1.66 & 0.700 \\
Lucile Ferreux & 41.5 & 2.21 & 1.62 & 3.01 & 0.000 \\
B S Shapiro & 22.7 & 0.81 & 0.66 & 1.00 & 0.055 \\
Total & 100 & 0.73 & 0.66 & 0.82 & 0.000 \\
\hline
\end{tabular}

Weight $=$ in $\%$, Odd. Ratio $=95 \%$ confidence limit, lower, Upper.

To assess the impact of the weight of the study, sensitivity analyses were performed by visual inspection of the forest plot displayed in chronicity of the study.

Data from four relevant articles were extracted and integrated into the meta-analysis. A meta-analysis of the six studies. Only English language and time restrictions 2001 and 2020. Relevant literature was also scanned through cross-references of identification in the reference lists within retrospective studies that fulfilled the following criteria, blastocyst, Day 5, Day 6, embryo transfer (E.T.) and pregnancy rate.

Forest plot (Figure 1), although some of the studies showed that transferring embryo on day 5 and day 6 are comparable but the forest plot indicates that the evidence of day 5 embryo transfer have a higher pregnancy rate with odd ratio and $95 \%$ confidence limit $0.73(0.66-0.82) p$ value $<0.000$.

The pregnancy rate was extracted and assigned as dichotomous frequency data. Then, the odds ratios with $95 \%$ confidence intervals calculated from individual studies before pooling, and the pooled odds ratios for investigated outcomes calculated using the random-effects model, considering that the underlying effects varied across the studies included. 


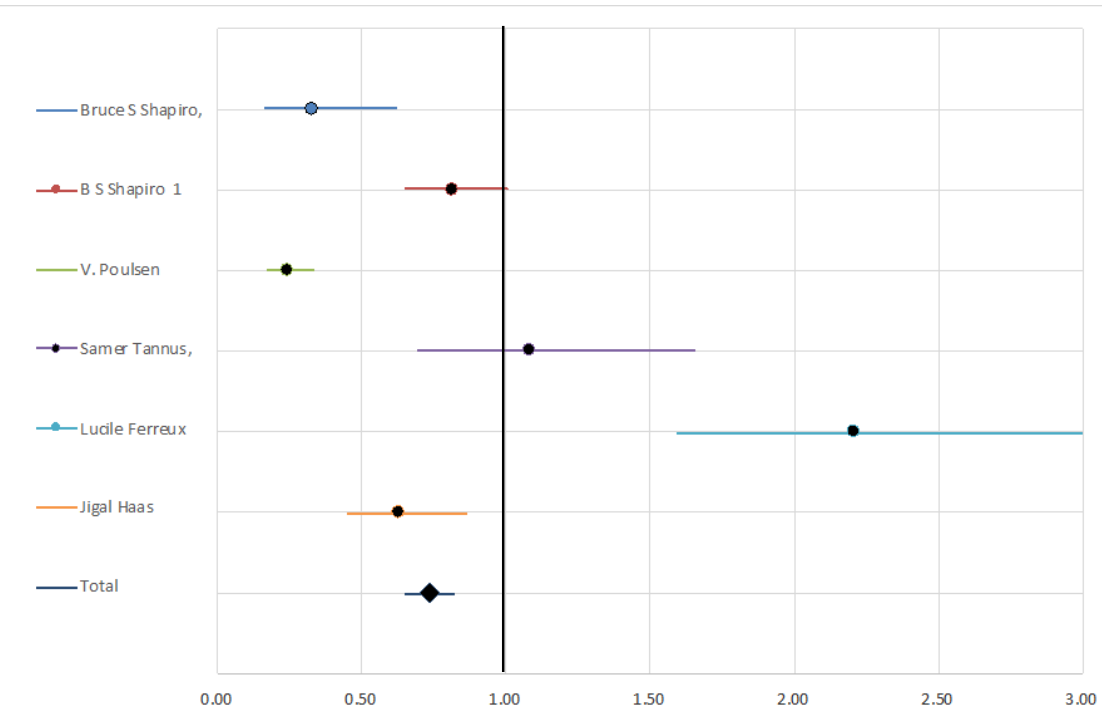

Figure 1. Forest plot of all the study with total.

Heterogeneity tests across the included studies conducted using I-squared and Q statistic. $P<0.010$ regarded as significant heterogeneity. Sensitivity analyses conducted to investigate outcomes and to assess the effects of a single study, from the overall analysis.

The inspection levels for pooled results are two-sided, and $P<0.05$ was considered to be statistically significant and SPSS (version 22.0) was used for all statistical analyses conducted in this study.

Literature search and study characteristics

Of the (211) potentially relevant studies screened by electronic databases, 191 excluded owing to irrelevant and duplicate topics. The full-text articles for the remaining 20 studies evaluated, and six articles met the inclusion criteria and included in this study (Figure 2).

\section{Results and the Role of Chance}

Data from six relevant articles were extracted and integrated into the meta-analysis. A meta-analysis of the six studies that reported clinical pregnancy rate (CPR) as an outcome, in 5640 cycles, 2274 cycle had embryo transfer at day 6 and the control was 3366 cycle had embryo transfer at day 5 showed a significantly higher clinical pregnancy rate following Day 5 embryo transfer compared with Day 6 embryo transfer with odd ratio and 95\% confidence limit 0.73 (0.66 0.82) $p$ value $<0.000[10]-[15]$.

\section{Discussion}

Data from previous studies, A prospective randomized controlled trial (Day 3 embryo transfer versus Day 5 blastocyst transfers) supporting embryo transfer day 3 and 5 with the efficiency of day 5 (blastocyst) is not inferior to that of day 3 (cleavage stage) [16]. 


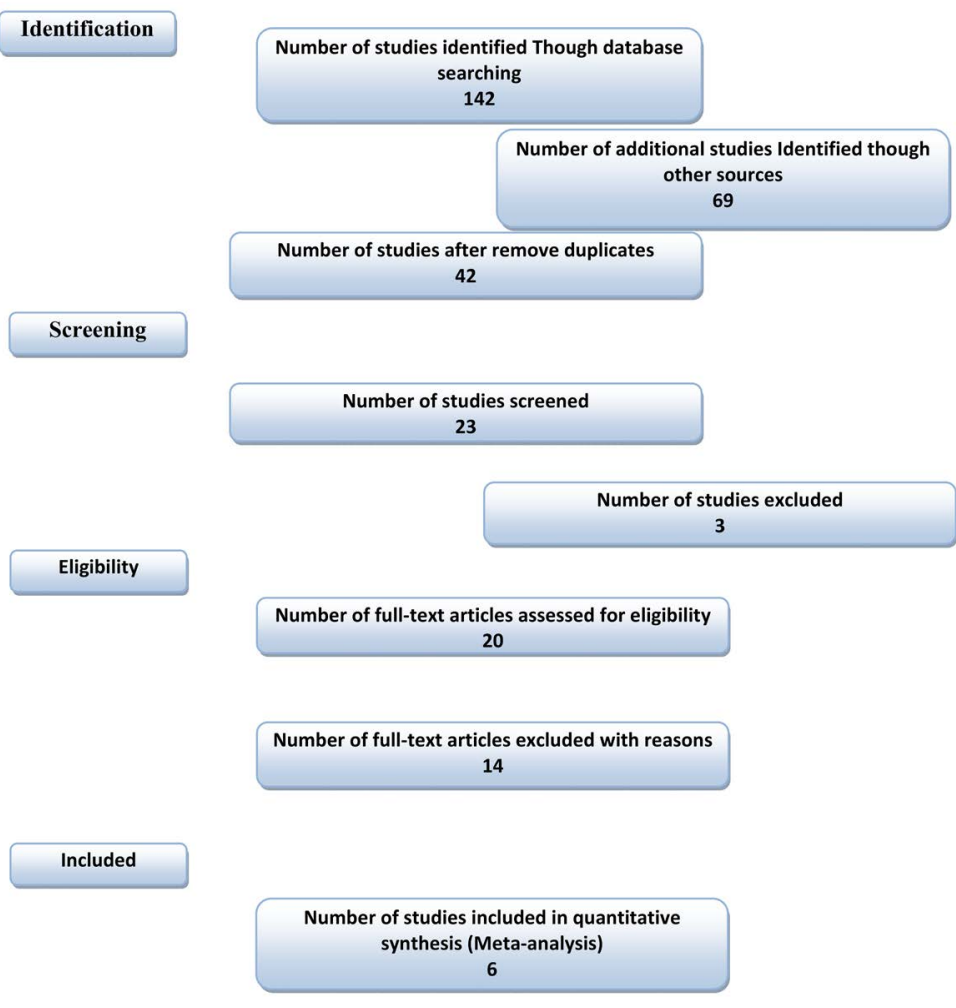

Figure 2. Presents the literature search in (Preferred Reporting Items for Systematic Reviews and Meta-Analysis statement) (PRISMA).

Recently embryo transfer day six becomes popular with a reasonable rate of implantation. Does day of embryo transfer really make a difference? An article concludes that embryo transfer at day six was independently associated with a significant decrease rate when compared to D5 transfer [17]. In systemic review and meta analysis, Day 5/6 ET continues to offer improved pregnancy rates without compromising birth outcomes [18].

In our study which is a meta-analysis, we concentrate on only clinical pregnancy rate as described, by most of the ART practitioners, should preferably transfer D5 rather than D6 blastocysts. Further RCTs are needed to address the question of whether D6 embryos should be transferred. It seems that it is difficult to conduct larger well-conducted studies of RCT, the number of studies is limited.

Current evidence shows that day 5 blastocysts embryo transfer is superior to Day 6. Due to the overall low quality of available evidence, larger and well-conducted studies are needed to compare the clinical pregnancy rate [13]. Other studies showed that clinical pregnancy rate was significantly higher following D5 compared to D6 BT [19].

A Cochrane Database of Systematic Reviews 2016, provides evidence that there is a small significant difference in live birth rates in favor of blastocyst transfer (Day 5 to day 6) [20]. The embryo quality, blastocyst expansion at D6 was independently associated with a significant decrease in clinical pregnancy rate compared to D5 expanded-blastocysts [14]. 


\section{Similar Results and Conclusions}

Clinical pregnancy rate was significantly higher following D5 compared to D6 BT [12]. The clinical pregnancy rate following embryo transfer is significantly lower with blastocysts on day 6 compared to blastocysts on day 5 [11]. Day 6 blastocysts transfer resulted in similar clinical pregnancy rates compared to day $5[20]$.

\section{Wider Implications of the Findings}

In regards to the results of this original meta-analysis, ART practitioners should preferably transfer D5 rather than D6 blastocysts. Further RCTs are needed to address the question of whether D6 embryos should be transfer.

\section{Contributors}

A.J., M.G. designed the study protocol and search, data extraction. AA and $\mathrm{HH}$ critical independent review with contributed to statistical analysis and interpretation of data. He, H. drafted the manuscript with $\mathrm{H} . \mathrm{H}$ critically revising the manuscript.

\section{Acknowledgements}

The authors would like to express their gratitude to all those who helped us during the writing of this manuscript (Mrs. Loreli Rayes, Miss Najwan AJ. Miss Ghaliah Aj and Miss Nouf Jarada). Appreciation to all the reviewers and editors for their view and idea

\section{Funding}

No funding.

\section{Limitations, Reasons for Caution}

The validity of meta-analysis results depends mainly on the quality and the number of published studies available. Indeed, this meta-analysis included no randomized controlled trial (RCT).

\section{Conflicts of Interest}

The authors declare no conflicts of interest regarding the publication of this paper.

\section{References}

[1] Bansal, K. (2011) Steps of IVF and ICSI. In: Bansal, K., Ed., Manual of Intrauterine Insemination (IUI) in Vitro Fertilization (IVF) and Intracytoplasmic Sperm Injection (ICSI), Jaypee Brothers Medical Publishers Pvt. Ltd., New Delhi, 68-68. https://doi.org/10.5005/jp/books/11209 15

[2] Yu, S., et al. (2018) New Application of Dydrogesterone as a Part of a Progestin-Primed Ovarian Stimulation Protocol for IVF: A Randomized Controlled Trial 
Including 516 First IVF/ICSI Cycles. Human Reproduction, 33, 229-237.

[3] D’Angelo, A., et al. (2019) Recommendations for Good Practice in Ultrasound: Oocyte Pick Up. Human Reproduction Open, 2019, hoz025.

https://doi.org/10.1093/hropen/hoz025

[4] Wang, C. and Swerdloff, R.S. (2014) Limitations of Semen Analysis as a Test of Male Fertility and Anticipated Needs from Newer Tests. Fertility and Sterility, 102, 1502-1507. https://doi.org/10.1016/j.fertnstert.2014.10.021

[5] Mullen, M.A. (2019) In Vitro Fertilization and Male-Factor Infertility. In: Burfoot, A., Ed., Encyclopedia of Reproductive Technologies, Routledge, London, 247-250. https://doi.org/10.4324/9780429037399-47

[6] Palermo, G.D., et al. (2017) Intracytoplasmic Sperm Injection: State of the Art in Humans. Reproduction, 154, F93-F110. https://doi.org/10.1530/REP-17-0374

[7] Bortoletto, P., Bakkensen, J. and Anchan, R.M. (2018) Embryo Transfer: Timing and Techniques. Minerva Endocrinologica, 43, 57-68.

[8] Shapiro, B.S., et al. (2001) A Comparison of Day 5 and Day 6 Blastocyst Transfers. Fertility and Sterility, 75, 1126-1130. https://doi.org/10.1016/S0015-0282(01)01771-X

[9] Moher, D., et al. (2009) Preferred Reporting Items for Systematic Reviews and Meta-Analyses: The PRISMA Statement. BMJ, 339, b2535.

https://doi.org/10.1136/bmj.b2535

[10] Siahaan, S. (2017) Pregnancy Rate on Blastocyst versus Cleavage Stage Embryo Transfer. The World Congress on Controversies in Obstetrics, Gynaecology and Infertility, Vienna, Austria, 30 November-2 December, 2017. https://doi.org/10.26226/morressier.59f838b6d462b8028d897311

[11] Haas, J., Meriano, J., Laskin, C., Bentov, Y., Barzilay, E., Casper, R.F., et al. (2016) Clinical Pregnancy Rate Following Frozen Embryo Transfer Is Higher with Blastocysts Vitrified on Day 5 than on Day 6. Journal of Assisted Reproduction and Genetics, 33, 1553-1557. https://doi.org/10.1007/s10815-016-0818-X

[12] Poulsen, V., et al. (2017) Elective Embryo Transfers on Day 6 Reduce Implantation Compared with Transfers on Day 5. Human Reproduction, 32, 1238-1243.

https://doi.org/10.1093/humrep/dex059

[13] Tannus, S., Cohen, Y. and Henderson, S. (2018) Fresh Transfer of Day 5 Slow-Growing Embryos versus Deferred Transfer of Vitrified, Fully Expanded Day 6 Blastocysts: Which Is the Optimal Approach? Human Reproduction, 34, 44-51. https://doi.org/10.1093/humrep/dey351

[14] Ferreux, L., Bourdon, M., Sallem, A., Santulli, P., Barraud-Lange, V., Foll, N.L., et al. (2018) Live Birth Rate Following Frozen-Thawed Blastocyst Transfer Is Higher with Blastocysts Expanded on Day 5 than on Day 6. Human Reproduction, 33, 390-398. https://doi.org/10.1093/humrep/dey004

[15] Sciorio, R., Thong, K. and Pickering, S.J. (2019) Increased Pregnancy Outcome after Day 5 versus Day 6 Transfers of Human Vitrified-Warmed Blastocysts. Zygote, 27, 279-284. https://doi.org/10.1017/S0967199419000273

[16] Hatırnaz, Ş. and KanatPektaş, M. (2017) Day 3 Embryo Transfer versus Day 5 Blastocyst Transfers: A Prospective Randomized Controlled Trial. Turkish Journal of Obstetrics and Gynecology, 14, 82-88. https://doi.org/10.4274/tjod.99076

[17] Maxwell, S.M., et al. (2015) A Comparison of Pregnancy Outcomes between Day 3 and Day 5/6 Embryo Transfers: Does Day of Embryo Transfer Really Make a Difference? Journal of Assisted Reproduction and Genetics, 32, 249-254. 
https://doi.org/10.1007/s10815-014-0404-Z

[18] Li, Y.X., Wang, J., Sun, T.Z., Lv, M.Q., Ge, P., Li, H.N., et al. (2020) Pregnancy Outcomes after Day 5 versus Day 6 Blastocyst-Stage Embryo Transfer: A Systematic Review and Meta-Analysis. Journal of Obstetrics and Gynaecology Research, 46, 595-605. https://doi.org/10.1111/jog.14188

[19] Bourdon, M., Pocate-Cheriet, K., Bantel, A.F.D., Grzegorczyk-Martin, V., Hoffet, A.A., Arbo, E., et al. (2019) Day 5 versus Day 6 Blastocyst Transfers: A Systematic Review and Meta-Analysis of Clinical Outcomes. Human Reproduction, 34, 1948-1964. https://doi.org/10.1093/humrep/dez163

[20] Glujovsky, D., Farquhar, C., Retamar, A.M.Q., Sedo, C.R.A. and Blake, D. (2016) Cleavage Stage versus Blastocyst Stage Embryo Transfer in Assisted Reproductive Technology. Cochrane Database of Systematic Reviews, No. 7, CD002118. https://doi.org/10.1002/14651858.CD002118.pub5 